\title{
Effect of MGO on inflammation in 3T3-L1 cells
}

\author{
A.L.M Vriends \\ Maastricht University \\ a.vriends@student.maastrichtuniversity.nl
}

\begin{abstract}
Advanced Glycation Endproducts (AGEs) play a major role in inducing adipose tissue inflammation associated with obesity-associated insulin resistance. MGO, a precursor for AGEs is accumulated during obesity. Therefore we hypothesized that increased MGO-derived AGEs induce inflammation in adipose tissue via the interaction with RAGE. Incubation of the $3^{T} 3-\mathrm{L} 1$ adipocytes with MGO-modified albumin increased the inflammatory gene expression. In addition, RAGE gene expression was increased and GLO-1 gene expression was decreased. So, inflammation is induced in $3^{T} 3^{-L} \mathrm{~L} 1$ adipocytes by MGO-derived AGEs after upregulation of RAGE gene expression. RAGE-mediated MGO-induced inflammation is an important mechanism involved in the obesity-induced adipose tissue inflammation.
\end{abstract}

\section{Keywords}

Advanced glycation end products, Inflammation, Methylglyoxal, obesity, Receptor for advanced glycation end products

\section{Introduction}

Overweight and obesity are growing public health problems and are associated with several adverse health consequences, which include type 2 diabetes, heart disease and cancer'. Besides the obesity epidemic, the rates of diabetes are increasing even more rapidly ${ }^{2}$. Research has demonstrated that inflammation in the obese adipose tissue is a very important link between these two conditions3. However, the exact danger signals underlying adipose tissue inflammation remain unclear. Advanced glycation endproducts (AGEs) play a major role in inducing adipose tissue inflammation. AGEs are stable endproducts of the glycation reaction ${ }^{4}$. Glycation is a non-enzymatic, post-translational modification of proteins, in which reducing sugars such as glucose, react with amino groups in proteins, lipids and nuclei acids through series of reactions producing AGEs ${ }^{5}$. This formation of AGEs, which is the result of a normal metabolism, proceeds over a long 
time and is a naturally occurring process. An increased formation of AGEs occurs in diverse settings such as obesity, diabetes and aging 4 .

Reactive dicarbonyl compounds, such as methylglyoxal (MGO), are AGE precursors and may react rapidly with proteins resulting in a very fast formation of specific AGEs within hours ${ }^{4,6}$. Therefore, MGO and MGO-derived AGEs are probably the most important players in AGE-related diseases. Preliminary data demonstrated that MGO levels are increased in adipose tissue of mice fed with a high fat diet. There is also shown that obese (Zucker) rats have higher concentrations of MGO in the adipose tissue compared to lean rats ${ }^{7.8}$.

MGO is naturally degraded by a specialized enzymatic mechanism, namely the glyoxalase system. This group of enzymes, consisted of two enzymes GLO-1 and GLO-2, catalyses the reaction of MGO into D-lactate 9 . There is recently shown that GLO-1 is locally decreased in most tissues during obesity 4,10 .

AGE precursors and AGEs can damage the cell trough diffusion. They modify circulating proteins in the blood such as albumin and bind and activate AGE receptors, like RAGE. This activtion lead to production of inflammatory cytokines and growth factors ${ }^{11}$.

RAGE, the receptor for AGEs is a pattern-recognition receptor, which is expressed at low levels in normal tissues and vasculature and becomes upregulated at sites where its ligands accumulate ${ }^{4,8}$. AGE-ligation to RAGE induces inflammation through persistent activation of the pro-inflammatory transcription factor NF-KB. Another downstream pathway activated trough binding from AGE on RAGE is the generation of reactive oxygen species (ROS) $)^{9,12}$. Recently, it is demonstrated that MGO-derived AGEs (MG- $\mathrm{H}_{1}$ ) are also very important ligands for RAGE ${ }^{13}$.

The aim of this study was to investigate whether MGO-derived AGEs can induce inflammation in adipose tissue by interaction with RAGE. This was studied by adding MGO-modified albumin to differentiated murine $3^{T} 3^{2}-L_{1}$ adipocytes during different time periods. Gene expression of inflammatory cytokines, RAGE and GLO-1 weas determined. We hypothesized that increased MGO-derived AGEs and decreased GLO-1 levels in obesity leads to adipose tissue inflammation which contributes to the development of type 2 diabetes. 


\section{Material and methods}

\section{Cell culture of murine 3T3-L1 cells}

Culture and differentiation from pre-adipocyt to mature adipocyte of the $3{ }^{T} 3$-L1 cells, which originally developed by clonal expansion from murine Swiss $3^{T} 3$ cells, was performed as described previously ${ }^{14,15}$.

\section{Incubation with MGO-albumin}

The biological effects of MGO-albumin were examined by incubating murine $3^{\mathrm{T} 3} \mathrm{~L}^{\mathrm{L} 1}$ adipocytes with $0.5 \mu \mathrm{M}$ control albumin, minimally and highly modified MGO-albumin for 6 hours and 24 hours. After incubation, total celullar RNA was extracted using Tri reagent (Sigma). Total RNA was reverse-transcribed with Iscript cDNA synthesis kit (Biorad) and expression of geses RAGE, GLO-1 and IL- 6 were measured quantitatively by real time PCR using SYBR Green mix (Quantace). The tresholdcycle (Ct) value for every sample was measured and mRNA experssion levels of each gene were determined by the $\Delta C$ method $^{16}$ using ß2-microglobulin and cyclophilin A as reference genes.

\section{Incubation with LPS and TNF-a}

3T3-L1 adipocytes were incubated with inflammatory stimuli to induce RAGE expression. The $3{ }^{T} 3-\mathrm{L} 1$ adipocytes were incubated with $10 \mathrm{ng} / \mu \mathrm{L}$ and $50 \mathrm{ng} / \mu \mathrm{L} \mathrm{LPS}$ or with $10 \mathrm{ng} / \mathrm{ml}$ TNF- $\alpha$ for 3, 6 and 18 hours. RNA isolation, cDNA synthesis and RT-PCR for the genes RAGE, GLO-1 and IL- 6 were performed as mentioned above.

Incubation with TNF-a and MGO-albumin

The biological effects of MGO-modified albumin after RAGE upregulation, was determined by pre-incubating murine $3^{T} 3$ - L1 adipocytes with $10 \mu \mathrm{g} / \mathrm{mL}$ TNF-a for 3 hours, followed by an incubation with $0.5 \mu \mathrm{M}$ control albumin, minimally and highly modified MGO-albumin for 6 hours and 24 hours. RNA isolation, CDNA synthesis and RT-PCR for the genes RAGE, GLO-1 PAI-1 and IL- 6 were performed as mentioned above.

\section{Statistics}

The statistical differences in gene expression levels of RAGE, IL- 6 and RAGE after incubation of 3T3-L1 cells with MGO, LPS or TNF-a were calculated using a unpaired Student's t-test. P-values less than 0.05 were considered statistically significant. All data are presented as mean $\pm S D$. 


\section{Results}

\section{Lipid accumulation after differentiation}

For determining the role of MGO-derived AGEs in inducing inflammation in the adipose tissue, an in vitro experiment was performed with murine 3T3-L1 adipocytes. Differentiation of pre-adipocytes to mature ${ }_{3}{ }^{T} 3$-L1 adipocytes is a model for adipogenesis. During this differentiation the adipocytes accumulate fat in vacuoles, which can be detected by Oilred-O staining (Figure 1).

A

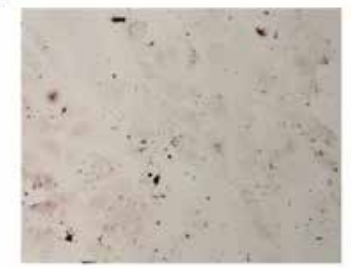

B

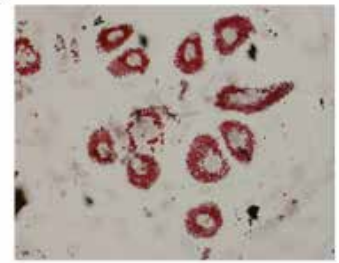

Figure 1. Intracellular lipid accumulation before (A) and after (B) differentiation

\section{MGO-derived AGEs did not induced inflammation in 3T3-L1 adipocytes}

There was no significant increase in gene expression of inflammatory cytokines after incubation with both MGO-induced modification (minimally and highly) of albumin (Figure $2 \mathrm{a}$ and $2 \mathrm{~b}$ ). In addition, the GLO-1 expression did not differ after both MGOmodified albumin incubation conditions (Figure $3 a$ and $3 b$ ).

A

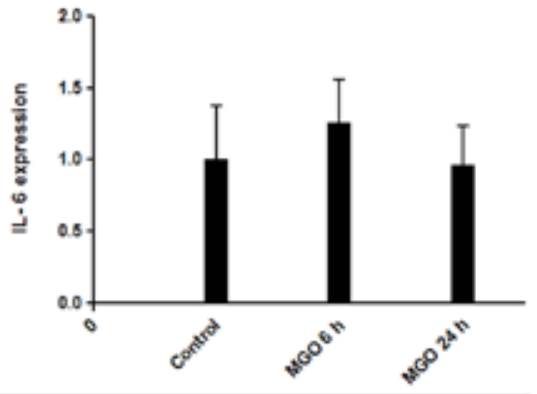

B

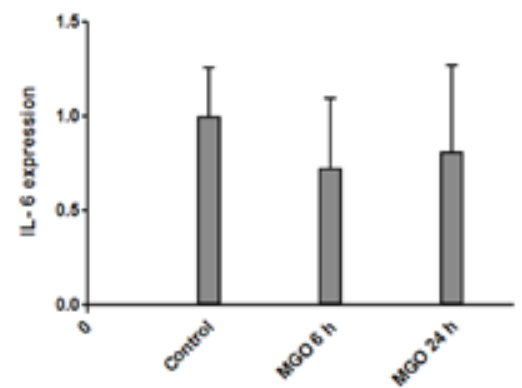

Figure 2. No difference of IL-6 expression after incubation with minimally and highly modified MGO. A) Incubation of murine 3T3-L1 adipocytes with control albumin or minimally modified MGO-albumin for 6 and 24 hour demonstrated no significant differences in IL- 6 gene expression. B) Incubation of murine 3T3-L1 adipocytes with control albumin or highly modified MGO-albumin for 6 and 24 hours exhibited no significant differences in IL- 6 gene expression. Data of all real time PCR are given as relative gene expression to the control albumin-treated condition and are normalized to the levels of ß2-microglobulin and cyclophilin A. Expression levels are presented as mean \pm SD. 
A

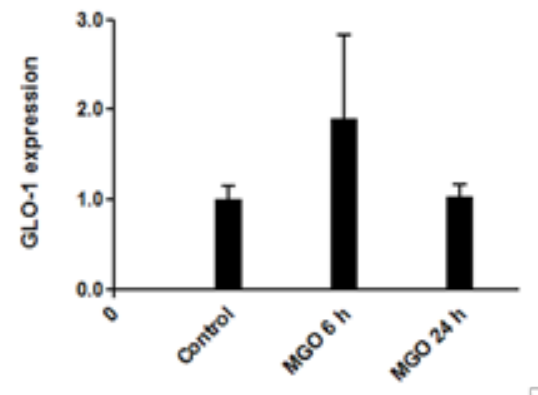

B

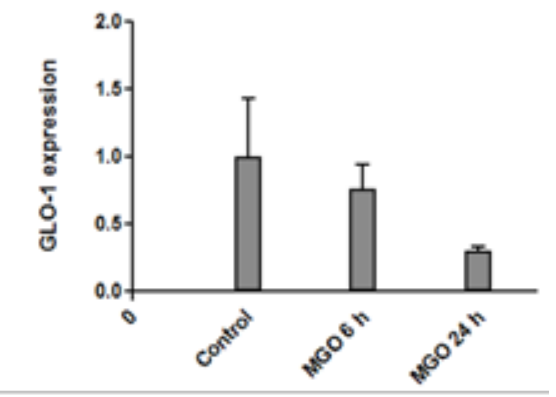

Figure 3. No difference of GLO-1 expression after incubation with minimally and highly modified MGO. A) 6 and 24 hour incubation of MGO-modified albumin to 3T3-L1 cells demonstrated no difference in GLO-1 gene expression. B) Highly modified MGO-albumin incubation to ${ }^{\mathrm{T}} \mathrm{T}$ - $\mathrm{L} 1$ cells demonstrated no-significant decrease of GLO-1 gene expression in time. Data of all real time PCR are given as relative gene expression to the control albumin-treated condition and are normalized to the levels of ß2-microglobulin and cyclophilin A. Expression levels are presented as mean \pm SD.

\section{Increased RAGE gene expression after 3 hour TNF-a stimulation}

Since no RAGE expression was seen after incubation with MGO-derived AGEs, we assume that the effects we observed (i.e. no effect on IL-6 and GLO-1 gene expression) were due to the lack of RAGE expression in 3T3-L1 adipocytes. Therefore, in order to stimulate the expression of RAGE in $3^{T} 3$-L1 adipocytes we first incubated the cells with different concentrations LPS and TNF-a for different time periods. All the stimulation conditions (10; $50 \mathrm{ng} / \mu \mathrm{L}$ LPS and $10 \mathrm{ng} / \mathrm{mL}$ TNF-a) and all the time periods (3, 6 and 18 hours) increased the IL- 6 gene experssion significantly. In addition, GLO-1 gene experssion was decreased after stimulation with both concentrations of LPS and the concentration TNF-a for all time periods. RAGE gene experssion was increased after 3-hour stimulation with both concentrations LPS and the concentration TNF- $a$, however only 3 hour stimulation with TNF-a resulted in an significant increase of RAGE gene experssion.

\section{MGO-derived AGEs induce inflammation in 3T3-L1 adipocytes}

To determine the role of AGEs in adipose tissue inflammation, $3^{\top} 3^{-L} \mathrm{~L} 1$ adipocytes were incubated with control albumin, minimally and highly modified albumin for different time periods. Before this incubation, the ${ }^{T} \mathrm{~T}_{3}-\mathrm{L} 1$ adipocytes were pre-incubated with $10 \mathrm{ng} / \mathrm{mL}$ TNF-a to increase the RAGE gene expression in this adipocytes. Incubation for 6 hours with minimally MGO-modified albumin induced a 6.0-fold increase of 
IL-6 gene expression compared to albumin-treated controls (Figure 4) after RAGE upregulation. A 24 hour incubation with minimally MGO-modified albumin increased significantly the IL-6 gene expression 1.9-fold ( $\mathrm{P}=0.043)$. Incubation with highly MGOmodified albumin for 6 and 24 hours increased the IL- 6 gene expression 1.5-fold and 2.4-fold respectively. The increase after $24 \mathrm{~h}$ was a significant increase $(P=0.015)$. 6 hour incubation with minimally MGO-modified albumin induced a significant 11.5fold increase of PAI-1 gene expression compared to albumin-treated controls after RAGE upregulation ( $\mathrm{P}<0.001$ ) (Figure 5). A 1.2-fold increase in PAl-1 gene expression was induced by an 24 hour incubation with minimally MGO-modified albumin. Incubation with highly MGO-modified albumin for 6 and 24 hours increased PAl-1 gene expression significant 13.3-fold and 2.4-fold respectively ( $P=0.007$ and $P=0.015$ resp.).

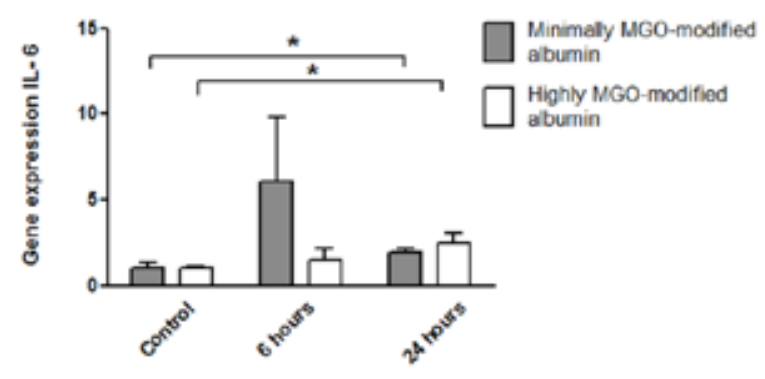

Figure 4. Increased IL-6 gene expression after TNF-a stimulation and incubation with minimally and highly modified MGO. Incubation with minimally MGO-modified albumin for 6 hours after TNF-a stimulation increased the IL- 6 gene expression not significant, whereas significance was reached after 24 hour incubation with minimally modified albumin. Highly MGO-modified albumin incubation for 6 hours and 24 increased IL- 6 gene expression, of which the increase after 24 hours was significant. Data of all real time PCR are given as relative gene expression to the control condition with no stimulation and are normalized to the levels of ß2-microglobulin and cyclophilin A. Expression levels are presented as mean \pm SD. * $p$-value $<0.05$ compared with control. 


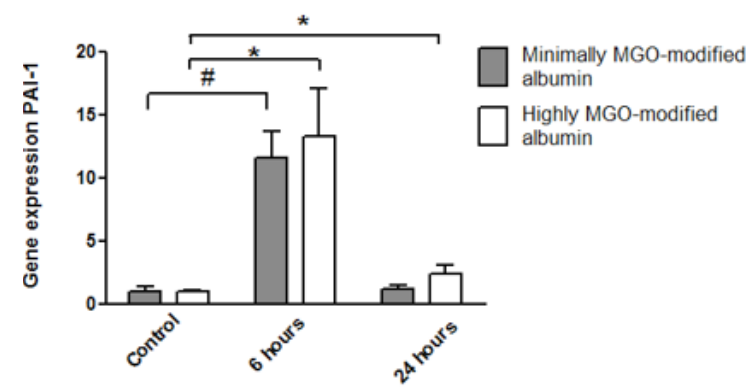

Figure 5. Increased PAl-1 gene expression after TNF-a stimulation and incubation with minimally and highly modified MGO. Incubation with minimally MGO-modified albumin for 6 hours after TNF-a stimulation increased the IL- 6 gene expression significant. 24 hour incubation increased PAI-1 gene expression not significant. Highly MGO-modified albumin incubation for 6 hours and 24 increased IL- 6 gene expression significant. Data of all real time PCR are given as relative gene expression to the control condition with no stimulation and are normalized to the levels of ß2-microglobulin and cyclophilin A. Expression levels are presented as mean \pm SD. ${ }^{*}$ p-value $<0.05$ compared with control. \#p<0.001 compared with control.

\section{MGO-derived AGEs decreased and increased GLO-1 gene expression in 3T3-L1 adipocytes}

A 6-hour incubation with minimally MGO-modified albumin significantly increased GLO-1 expression 2.9-fold after RAGE upregulation ( $P=0.013$ ) (Figure 6). Incubation for 24 hours decreased GLO-1 gene expression 0.7-fold. A significant 0. 6-fold decrease in GLO-1 expression was reached with incubation of highly MGO-modified albumin for 6 hours $(P=0.037)$. An incubation of 24 hours had no significant effect on the gene expression of GLO-1.

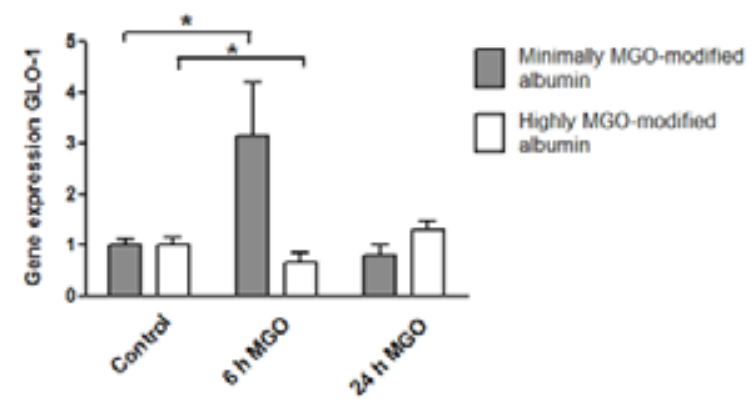

Figure 6. Effect of minimally and highly MGO-modified albumin incubation on GLO-1 gene expression. Incubation with minimally MGO-modified albumin for 6 hours after TNF-a stimulation increased the GLO-1 expression significant. 24 hour incubation decreased GLO-1 gene expression not significant. Highly MGOmodified albumin incubation for 6 hours decreased GLO-1 expression significant, whereas 24 hour incubation had no significant effect on GLO-1 gene expression. Data of all real time PCR are given as relative gene expression to the control condition with no stimulation and are normalized to the levels ß2-microglobulin and cyclophilin A. Expression levels are presented as mean \pm SD. ${ }^{*}$ p-value <0.05 compared with control. 


\section{MGO-derived AGEs increase RAGE expression in 3T3-L1 adipocytes}

Stimulation with TNF-a followed by an incubation with minimally MGO-modified albumin for 6 and 24 hours resulted in a 2.3-fold and 1.4-fold increase of RAGE gene expression respectively (Figure 7). The increase of gene expression after 6 hours was significant $(P=0.009)$. An incubation of 6 hours with highly MGO-modified albumin induced a significant 1.8-fold increase of RAGE gene expression ( $P=0.031)$, whereas an 24 hour incubation had no significant effects on the RAGE gene expression.

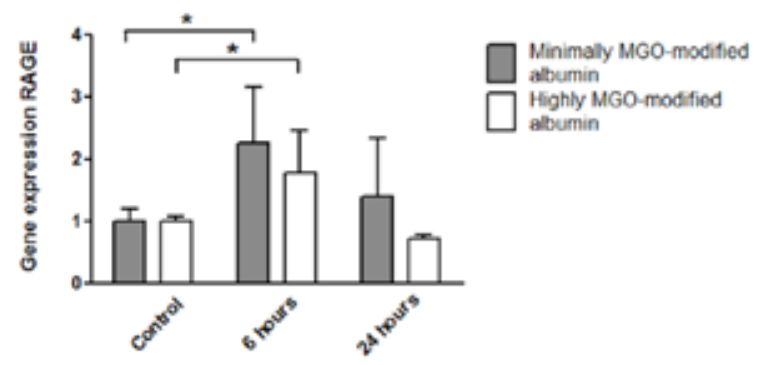

Figure 7. Increase in RAGE expression after TNF-a stimulation and incubation with minimally and highly MGO-modified albumin. Incubation with minimally MGO-modified albumin for 6 hours after TNF-a stimulation increased the RAGE gene expression significant. 24 hour incubation increased RAGE gene expression not significant. Highly MGO-modified albumin incubation for 6 hours increased RAGE expression significant, whereas 24 hour incubation has no significant effect on RAGE gene expression. Data of all real time PCR are given as relative gene expression to the control condition with no stimulation and are normalized to the levels ß2-microglobulin and cyclophilin A. Expression levels are presented as mean \pm SD. * p-value <0.05 compared with control.

\section{Conclusion}

In this study we have shown that MGO-derived AGEs induced inflammatory markers in cultured 3T3-L1 adipocytes. RAGE gene expression was upregulated through MGO-derived AGEs, whereas both an increase and decrease of GLO-1 gene expression was observed. This AGE-induced inflammation can be a key player in the adipose tissue inflammation observed in the pathophysiology of obesity-related complications. Further research have to show the effect of MGO-incubation on protein levels and if this effect is present in human cells. In addition, RAGE antibodies have to elucidate if MGO-induced inflammation is caused by RAGE. Finally, the effect of MGO on insulin signalling have to be examined. 


\section{Role of the student}

Anne Vriends was an ungraduated student working under the supervision of Katrien Gaens when the research in this report was performed. The topic and study design was proposed by the supervisor. Performing the experiments, formulating of the conclusions and the writing were done by the student.

\section{References}

1. Abelson P, Kennedy D. The obesity epidemic. Science (New York, NY). 2004;304(5676):1413.

2. Seidell JC. Obesity, insulin resistance and diabetes-a worldwide epidemic. British Journal of Nutrition. $2000 ; 83\left(S_{1}\right): S_{5}-S 8$

3. Dandona P, Aljada A, Bandyopadhyay A. Inflammation: the link between insulin resistance, obesity and diabetes. Trends in immunology. 2004;25(1):4-7.

4. Gaens KH, Stehouwer CD, Schalkwijk CG. Advanced glycation endproducts and its receptor for advanced glycation endproducts in obesity. Current opinion in lipidology. 2013;24(1):4-11.

5. Singh R, Barden A, Mori T, Beilin L. Advanced glycation end-products: a review. Diabetologia. 2001;44(2):129-46.

6. Thornalley P, Langborg A, Minhas H. Formation of glyoxal, methylglyoxal and 3-deoxyglucosone in the glycation of proteins by glucose. Biochem J. 1999;344:109-16.

7. Jia X, Chang T, Wilson TW, Wu L. Methylglyoxal mediates adipocyte proliferation by increasing phosphorylation of Akt1. PloS one. 2012;7(5):e36610.

8. Jia X, Wu L. Accumulation of endogenous methylglyoxal impaired insulin signaling in adipose tissue of fructose-fed rats. Molecular and cellular biochemistry. 2007;306(1-2):133-9.

9. Rabbani N, Thornalley PJ. Methylglyoxal, glyoxalase 1 and the dicarbonyl proteome. Amino acids. 2012;42(4):1133-42.

10. Rabbani N, Thornalley PJ, editors. Glyoxalase in diabetes, obesity and related disorders. Seminars in cell \& developmental biology; 2011: Elsevier.

11. Brownlee M. The pathobiology of diabetic complications a unifying mechanism. Diabetes. 2005;54(6):1615-25.

12. Matafome P, Sena C, Seiça R. Methylglyoxal, obesity, and diabetes. Endocrine. 2013;43(3):472-84.

13. Xue J, Ray R, Singer D, Böhme D, Burz DS, Rai V, et al. The receptor for advanced glycation end products (RAGE) specifically recognizes methylglyoxal-derived AGEs. Biochemistry. 2014;53(20):3327-35.

14. Student AK, Hsu R, Lane M. Induction of fatty acid synthetase synthesis in differentiating $3{ }^{T} 3-L_{1}$ preadipocytes. Journal of Biological Chemistry. 1980;255(10):4745-50.

15. Zebisch K, Voigt V, Wabitsch M, Brandsch M. Protocol for effective differentiation of $3 \mathrm{~T}^{\mathrm{T}}$-L1 cells to adipocytes. Analytical biochemistry. 2012;425(1):88-90.

16. Schmittgen TD, Livak KJ. Analyzing real-time PCR data by the comparative CT method. Nature protocols. 2008;3(6):1101-8. 